Original paper

\title{
National dosimetric audit network finds discrepancies in AAA lung inhomogeneity corrections
}

\author{
Leon Dunn ${ }^{\text {a, }}$, , Joerg Lehmann ${ }^{\text {a, b, e }}$, Jessica Lye ${ }^{\text {a }}$, John Kenny ${ }^{\text {a, c }}$, Tomas Kron ${ }^{\text {e, }}$, \\ Andrew Alves ${ }^{\mathrm{a}}$, Andrew Cole ${ }^{\mathrm{a}, \mathrm{d}}$, Jackson Zifodya ${ }^{\mathrm{g}}$, Ivan Williams ${ }^{\mathrm{a}, \mathrm{e}}$ \\ a Australian Clinical Dosimetry Service, Yallambie, Victoria 3085, Australia \\ ${ }^{\mathrm{b}}$ Institute of Medical Physics, University of Sydney, Sydney, Australia \\ ${ }^{\mathrm{c}}$ Epworth Radiation Oncology, Richmond, Victoria 3121, Australia \\ d Australian Radiation Protection and Nuclear Safety Agency, Yallambie, Victoria 3085, Australia \\ e School of Applied Science, RMIT University, Melbourne, Australia \\ ${ }^{\mathrm{f}}$ Peter MacCallum Cancer Centre, Melbourne 3008, Australia \\ ${ }^{g}$ North West Cancer Centre, Tamworth Hospital, Tamworth, NSW, Australia
}

\section{A R T I C L E I N F O}

\section{Article history:}

Received 11 January 2015

Received in revised form

1 April 2015

Accepted 2 April 2015

Available online 23 April 2015

\section{Keywords:}

Audit

Dosimetry

Radiotherapy

Algorithm

Dose

\begin{abstract}
A B S T R A C T
This work presents the Australian Clinical Dosimetry Service's (ACDS) findings of an investigation of systematic discrepancies between treatment planning system (TPS) calculated and measured audit doses. Specifically, a comparison between the Anisotropic Analytic Algorithm (AAA) and other common dose-calculation algorithms in regions downstream $(\geq 2 \mathrm{~cm})$ from low-density material in anthropomorphic and slab phantom geometries is presented. Two measurement setups involving rectilinear slabphantoms (ACDS Level II audit) and anthropomorphic geometries (ACDS Level III audit) were used in conjunction with ion chamber (planar 2D array and Farmer-type) measurements. Measured doses were compared to calculated doses for a variety of cases, with and without the presence of inhomogeneities and beam-modifiers in 71 audits. Results demonstrate a systematic AAA underdose with an average discrepancy of $2.9 \pm 1.2 \%$ when the AAA algorithm is implemented in regions distal from lung-tissue interfaces, when lateral beams are used with anthropomorphic phantoms. This systemic discrepancy was found for all Level III audits of facilities using the AAA algorithm. This discrepancy is not seen when identical measurements are compared for other common dose-calculation algorithms (average discrepancy $-0.4 \pm 1.7 \%$ ), including the Acuros XB algorithm also available with the Eclipse TPS. For slab phantom geometries (Level II audits), with similar measurement points downstream from inhomogeneities this discrepancy is also not seen.

Crown Copyright $\odot 2015$ Published by Elsevier Ltd on behalf of Associazione Italiana di Fisica Medica. This is an open access article under the CC BY-NC-SA license (http://creativecommons.org/licenses/bync-sa/4.0/).
\end{abstract}

\section{Introduction}

In radiation therapy treatment planning, the dose calculation algorithm of the treatment planning system (TPS) is relied upon to accurately calculate the dose and determine the Monitor Units (MU) to be delivered by the treatment linear accelerator (linac) to treat a particular tumour. Commercial treatment planning systems each use a particular 'brand' of algorithm [1]. The major (non-full Monte Carlo) dose calculation algorithms used in TPS are:

\footnotetext{
* Corresponding author. Tel.: +61 424907146.

E-mail address: leon.dunn@austin.org.au (L. Dunn).
}

- Superposition [2]

- Collapsed Cone Convolution [3] (CCC)

- Anisotropic Analytical Algorithm [4] (AAA

- Pencil Beam [5,6] (PB)

- Collapsed Cone [7] (CC)

Generally, dose calculation algorithms fall into correction-based equivalent path-length algorithms and model based algorithms [8] with electron transport either explicitly accounted for, or neglected. There is a large body of literature evaluating the performance of each algorithm both compared to other algorithms/Monte Carlo [8-12] in clinical scenarios and measurement [13-15]. It is known that each algorithm has its associated advantages and disadvantages in terms of speed of calculation, accuracy and in dealing with 
inhomogeneities in both tissue structure and density. Improvements in the accuracy of calculated doses have been shown when algorithms explicitly consider volume scatter and changes in electron transport in low/high density regions, such as lung or bone.

In Australia, the Australian Clinical Dosimetry Service (ACDS) [16] has been performing Level II [17] and Level III audits [18] across Australian radiotherapy facilities for the past two years. In both audits, measurements are compared to doses calculated with the algorithms listed above for both anthropomorphic (Level III) and slab geometries (Level II). Measurements for both audits are done onsite by an ACDS audit team using Primary Standards Laboratory (PSL) calibrated Farmer type ionization chambers and an array of ionization chambers. To date (2014), 36 Level II and 35 Level III audits have been performed evaluating various algorithms. Gathering such a large amount of data has allowed the ACDS to begin identifying systematic, consistent calculation discrepancies between algorithms for particular phantom measurements. Such a discrepancy is the topic of this paper.

The AAA dose calculation model is a 3D pencil beam convolution superposition algorithm that has separate modelling for primary photons, scattered extra-focal photons, and electrons scattered from the beam limiting devices [19,20]. The AAA algorithm is convolution based with the dose from each pencil beam, or beamlet, being calculated through a convolution. Each beamlets' energy fluence is separated into components for primary and extrafocal photons and electron contamination. The dose distribution $D_{\beta}(x, y, z)$ for beamlet $\beta$ is calculated using Equation (1) [21,22].

$D_{\beta}(x, y, z)=\varphi_{\beta} \times I_{\beta}(z, \rho) \times \iint_{\beta} K_{\beta}\left(x^{\prime}-x, y^{\prime}-y, z, \rho\right) d x^{\prime} d y^{\prime}$

Here, the dose is calculated by a convolution of $\phi_{\beta}$, the beamlet's fluence, $I_{\beta}$, an energy deposition function and a scatter kernel $K_{\beta}(x, y, z, \rho)$ which defines the lateral scatter component of the dose. The scatter kernels are density dependent and calculated in multiple directions laterally around the beamlet. The total dose calculated in a patient is a result of a superposition of each beamlet's contribution.

This paper presents an investigation into systematic discrepancies between TPS calculated and measured audit doses seen when comparing the AAA algorithm's prediction to other algorithms, in regions adjacent to and downstream from low-density material in anthropomorphic and slab phantom geometries.

\section{Materials and methods}

\section{Level III (WAIL) audit}

The ACDS Level III Audit [18] determines absorbed dose to water delivered to selected points in an anthropomorphic phantom (CIRS IMRT Phantom Model 002LFC (thorax), Computerized Imaging Reference Systems, Inc., Norfolk, VA, United States). An axial CT slice of the phantom is shown in Fig. 1. The Level III audit is an "end-toend" test of the complete radiotherapy treatment chain. The Level III audit includes Wedged and Asymmetric fields with Inhomogeneities in Lung (WAIL). For this audit, five ACDS prescribed treatment cases are planned and the dose to selected points, indicated by numbers in Fig. 1, is calculated. Treatment fields are fully prescribed by the ACDS using 3D conformal planning for $6 \mathrm{MV}$ photons only with static gantry and collimation. The details of the five cases are shown in Table 1 . Cases 2 and 3 are based on cases 2 and 7 from IAEA TECDOC 1583 [23], while the others have been developed by the ACDS as reference (Case 1 ) and to further investigate the impact of the wedge (Cases 4 and 5 , added later).

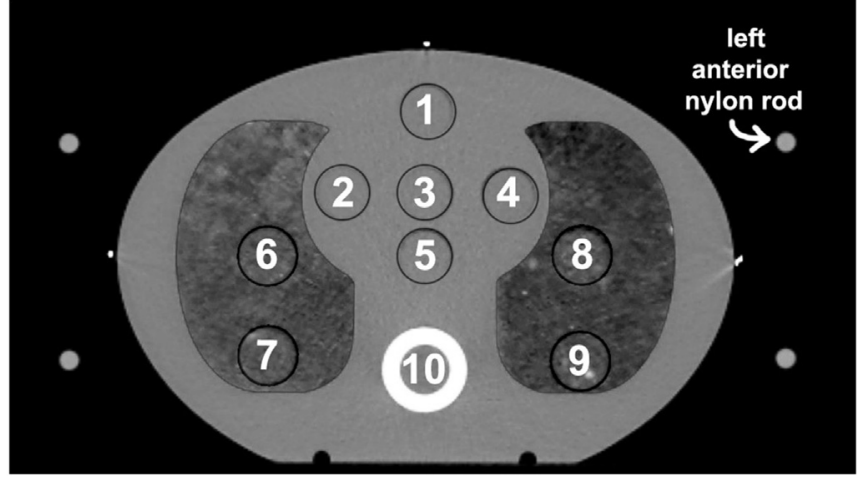

Figure 1. A sample CT of the central slice of the Level III phantom including the measurement points used in the ACDS Level III audit.

Dosimetry measurements are made by an onsite audit team with PSL calibrated Farmer type ionisation chambers and electrometers. Measurement locations correspond to the selected calculated points, which are listed for each case in Table 1 Fig. 1.

To investigate the findings of this study with the intent of finding a practical solution for facilities using the Eclipse AAA dose calculation algorithm, additional dose calculations were performed using the Acuros XB algorithm, which is also available with the Eclipse TPS, (Varian Medical Systems, Palo Alto, CA). Acuros XB is a modern algorithm which calculates dose to water by directly solving the Linear Boltzmann Transport Equation (LBTE). The algorithm can also calculate dose-to-medium, however for comparison to the current generation of algorithms evaluated in this study, only dose to water calculations are presented. Using a CT scan of the Level III phantom from one facility, absorbed doses per MU were calculated with AAA and Acuros for all measured points discussed.

\section{Level II audit}

The ACDS Level II Audit [17] evaluates accuracy of absorbed dose to water delivered to selected arrays of points at specific planes within a "slab" geometry phantom. This is an audit of the beam model within a treatment planning system, where the simulated (computer generated) CT of the measurement phantom is supplied by the ACDS to the Facility for treatment planning. The Level II audit includes Wedged and Asymmetric fields with Inhomogeneities for Lung (WAIL) treatments with sixteen separate single field cases planned. Fields are fully prescribed by the ACDS and underpin the ACDS Level III (WAIL) Audit. The audit is for 3D planning with $6 \mathrm{MV}$ photons only with static (zero degree) gantry and collimator angles. Centres audited planned using their own treatment planning systems with an ACDS supplied CT to ED (electron density) table.

Dosimetry measurements are made in custom phantoms of CIRS Solid Water and CIRS Lung materials (Computerized Imaging Reference Systems, Inc., Norfolk, VA, United States). The detector used in the Level II audit is a PTW Octavius 729 2D ionisation chamber array (PTW GmbH, Freiburg Germany). Measurements are performed at two different depths for each field from Case 4-16. Details of the 16 cases are included in Table 2. Case 1 is not listed in this table since it is not measured. Case 1 is planned only and constitutes a $10 \times 10 \mathrm{~cm}^{2}$ field with $10 \mathrm{~Gy}$ prescribed to the dose output specification point of the facility. This is used to verify that the treatment planning system calculates $1000 \pm 5 \mathrm{MU}$ to be delivered, as by definition the dose to the dose output specification point is $1 \mathrm{cGy} / \mathrm{MU}$.

The computer generated CT series (ImSimQA, version 3.0.76a, Oncology Systems Ltd, Delmar, NY) used in the Level II WAIL Audit are based on a $30 \times 30 \times 30 \mathrm{~cm}^{3}$ cube of water. Series 1 consists of 
Table 1

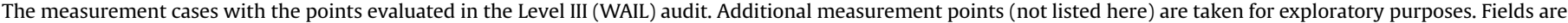

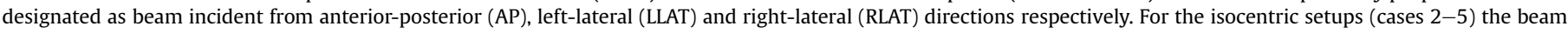
setup point is specified in parentheses. Further specific details on beam arrangements can be found in Ref. [18].

\begin{tabular}{|c|c|c|c|c|c|}
\hline Case & Field & Measurement points & Setup & Field size & Wedge \\
\hline 1 & $\mathrm{AP}$ & 1,10 & $\mathrm{SSD}=100 \mathrm{~cm}$ & $10 \mathrm{~cm}(\mathrm{SI}) \times 10 \mathrm{~cm}(\mathrm{LR})$ & None \\
\hline 2 & LLAT & 1,4 & Isocentric (Point 1) & $8 \mathrm{~cm}(\mathrm{SI}) \times 10 \mathrm{~cm}(\mathrm{AP})$ & $45^{\circ}$ wedge \\
\hline 3 & AP & $4,5,10$ & Isocentric (Point 3) & $10 \mathrm{~cm}(\mathrm{SI}) \times 12 \mathrm{~cm}(\mathrm{LR})$ & None \\
\hline 3 & LLAT & 5 & Isocentric (Point 3) & $10 \mathrm{~cm}(\mathrm{SI}) \times 0.6 \mathrm{~cm}(\mathrm{AP})$ & $30^{\circ}$ wedge \\
\hline 3 & RLAT & 5 & Isocentric (Point 3) & $10 \mathrm{~cm}(\mathrm{SI}) \times 0.6 \mathrm{~cm}(\mathrm{AP})$ & $30^{\circ}$ wedge \\
\hline 4 & LLAT & $2,3,4,5$ & Isocentric (Point 3) & $10 \mathrm{~cm}(\mathrm{SI}) \times 12 \mathrm{~cm}(\mathrm{AP})$ & None \\
\hline 5 & LLAT & $2,3,4,5$ & Isocentric (Point 3) & $10 \mathrm{~cm}(\mathrm{SI}) \times 12 \mathrm{~cm}(\mathrm{AP})$ & $60^{\circ}$ wedge \\
\hline
\end{tabular}

Table 2

The cases used in the Level II (WAIL) audit.

\begin{tabular}{|c|c|c|c|c|c|}
\hline CT series & Case & Measurement depths $(\mathrm{cm})$ & $\mathrm{SSD}(\mathrm{cm})$ & Field size & Wedge \\
\hline 1 & 2 & 10 & 90 & $20 \mathrm{~cm}(\mathrm{SI}) \times 20 \mathrm{~cm}(\mathrm{LR})$ & None \\
\hline 1 & 3 & 10 & 90 & $10 \mathrm{~cm}(\mathrm{SI}) \times 10 \mathrm{~cm}(\mathrm{LR})$ & None \\
\hline 1 & 4 & 8,15 & 85 & $12 \mathrm{~cm}(\mathrm{SI}) \times 12 \mathrm{~cm}(\mathrm{LR})$ & None \\
\hline 1 & 5 & 8,15 & 85 & $12 \mathrm{~cm}(\mathrm{SI}) \times 12 \mathrm{~cm}(\mathrm{LR})$ & $30^{\circ}$ wedge \\
\hline 1 & 6 & 8,15 & 85 & $8 \mathrm{~cm}(\mathrm{SI}) \times 10 \mathrm{~cm}(\mathrm{LR})$ & None \\
\hline 1 & 7 & 8,15 & 85 & $8 \mathrm{~cm}(\mathrm{SI}) \times 10 \mathrm{~cm}(\mathrm{LR})$ & $30^{\circ}$ wedge \\
\hline 1 & 8 & 8,15 & 85 & $10 \mathrm{~cm}(\mathrm{SI}) \times 0,6 \mathrm{~cm}(\mathrm{LR})$ & None \\
\hline 1 & 9 & 8,15 & 85 & $10 \mathrm{~cm}(\mathrm{SI}) \times 0,6 \mathrm{~cm}(\mathrm{LR})$ & $60^{\circ}$ wedge \\
\hline 1 & 10 & 8,15 & 85 & $4 \mathrm{~cm}(\mathrm{SI}) \times 4 \mathrm{~cm}(\mathrm{LR})$ & None \\
\hline 2 & 11 & 8,15 & 85 & $12 \mathrm{~cm}(\mathrm{SI}) \times 12 \mathrm{~cm}(\mathrm{LR})$ & None \\
\hline 2 & 12 & 8,15 & 85 & $12 \mathrm{~cm}(\mathrm{SI}) \times 12 \mathrm{~cm}(\mathrm{LR})$ & $30^{\circ}$ wedge \\
\hline 2 & 13 & 8,15 & 85 & $8 \mathrm{~cm}(\mathrm{SI}) \times 10 \mathrm{~cm}(\mathrm{LR})$ & None \\
\hline 2 & 14 & 8,15 & 85 & $8 \mathrm{~cm}(\mathrm{SI}) \times 10 \mathrm{~cm}(\mathrm{LR})$ & $30^{\circ}$ wedge \\
\hline 3 & 15 & 8,15 & 85 & $10 \mathrm{~cm}(\mathrm{SI}) \times 0,6 \mathrm{~cm}(\mathrm{LR})$ & None \\
\hline 3 & 16 & 8,15 & 85 & $10 \mathrm{~cm}(\mathrm{SI}) \times 0,6 \mathrm{~cm}(\mathrm{LR})$ & $60^{\circ}$ wedge \\
\hline
\end{tabular}

only water. For Series 2 and 3 a $5 \mathrm{~cm}$ thick slab of inhale lung is placed within the cube at a depth of $1 \mathrm{~cm}$ (to boundary). The central transverse slices of the three CT series are shown in Fig. 2. Dose calculations are performed by the facilities on a $1 \mathrm{~mm}^{3}$ dose grid.

\section{Results}

Performance of the AAA algorithm in the level III audit

In Fig. 3 the results from the Level III audit are shown for all 12 points downstream from a low density (lung) inhomogeneity; referring to Table 1 and Fig. 1 these points are: Case 2 Points 1 and 4 , Case 3 Point 5 for left and right lateral, Case 4 all points, and Case 5 all points. Each point shows the dose difference/deviation from ACDS [(planned dose - measured dose)/(measured dose)] relative to the dose difference of the reference Case 1 Point 1 to eliminate daily linac output and individual facility dosimetry deviations. Figure 3 (a) shows the results of audits of facilities using Eclipse AAA and (b) shows the results of audits using all facilities using other algorithms. The average difference $( \pm 1 \sigma)$ for all the points calculated with the AAA algorithm is $2.9 \pm 1.2 \%$ high (Fig. 3a). This means, following the above given ACDS definition of difference that the treatment linacs were underdosing. The average difference and standard deviation for the points calculated with the other algorithms is $-0.4 \% \pm 1.7 \%$ (Fig. $3 b$ ).

In Fig. 4 the average audit results are shown for individual points downstream from the lung inhomogeneity. Again, all results have been normalized to the corresponding reference (Case 1, Point 1) to eliminate linac output and individual facility dosimetry deviations. As before, the results are divided into audits with the AAA algorithm and audits with all other algorithms. The 3.0\% underdose is present consistently with all points behind lung when using the AAA algorithm, regardless of the presence of a wedge, depth behind lung, or distance to field edge.

A comparison of calculations with the AAA algorithm to calculations done with the Acuros algorithm (dose to water) in the same phantom and planning system are shown in Fig. 5. The diagram shows differences in dose per MU for the same points in the

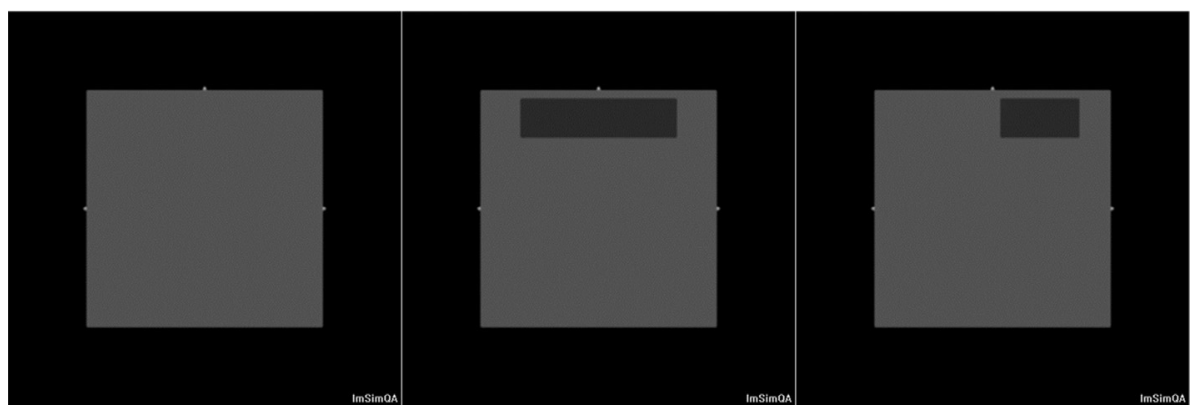

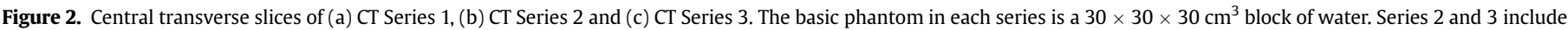
volumes of material equivalent to "inhale" lung tissue. 

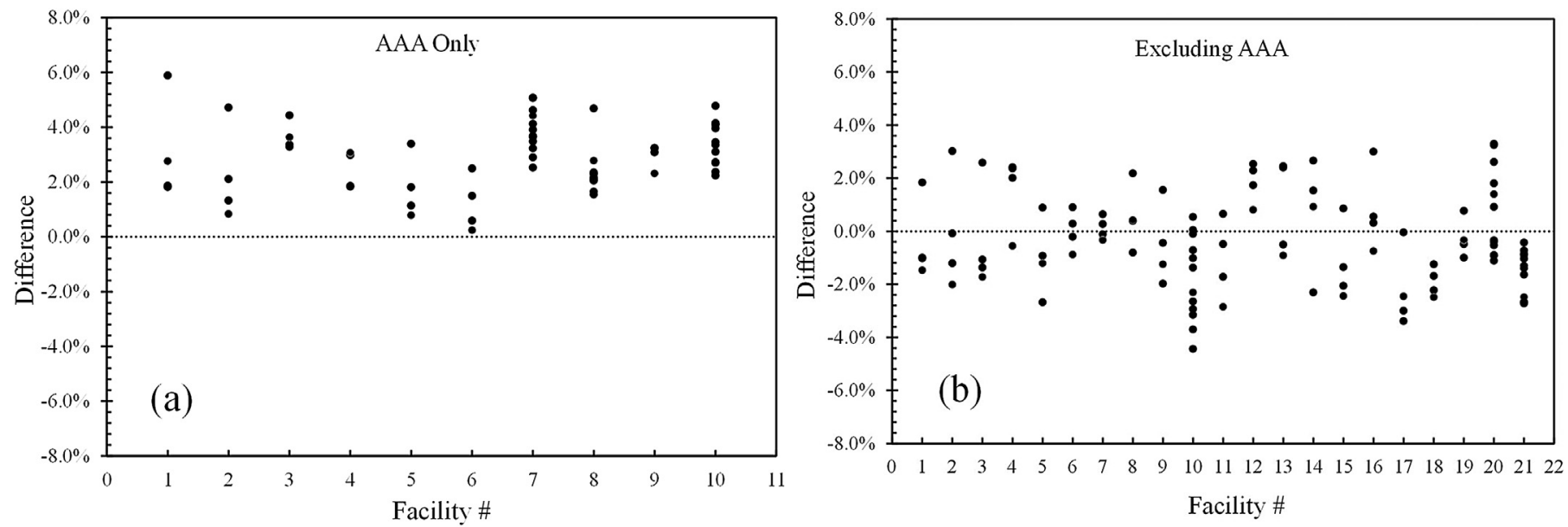

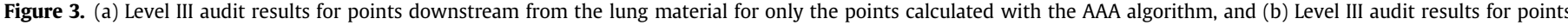

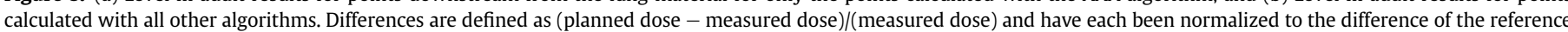

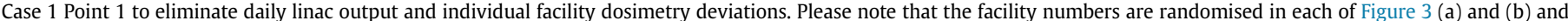
Facilities $1-11$ do not correspond to the same facilities for both audits.

phantom as analysed before. The difference on average was found to be $-2.8 \%$ and thereby generally of the same magnitude and in the opposite direction as the deviation found for the AAA algorithm calculations in comparison to the measurements across the country. The exception is Case 2 Point 4, where the difference is smaller but still in the same direction with $-0.85 \%$. These results indicate that the Acuros $\mathrm{XB}$ algorithm largely corrects the erroneous calculation by the AAA algorithm.

\section{Performance of the AAA algorithm in the level II audit}

The Level II results have been analysed and are presented in the same manner as the Level III results (Figs. 3 and 4). Figure 5 (a) shows the Level II audit results for all facilities evaluated in the cases where the measurement point was downstream from the lung inhomogeneity using the AAA algorithm. Figure 6 (b) shows the Level II audit results for all facilities excluding those facilities

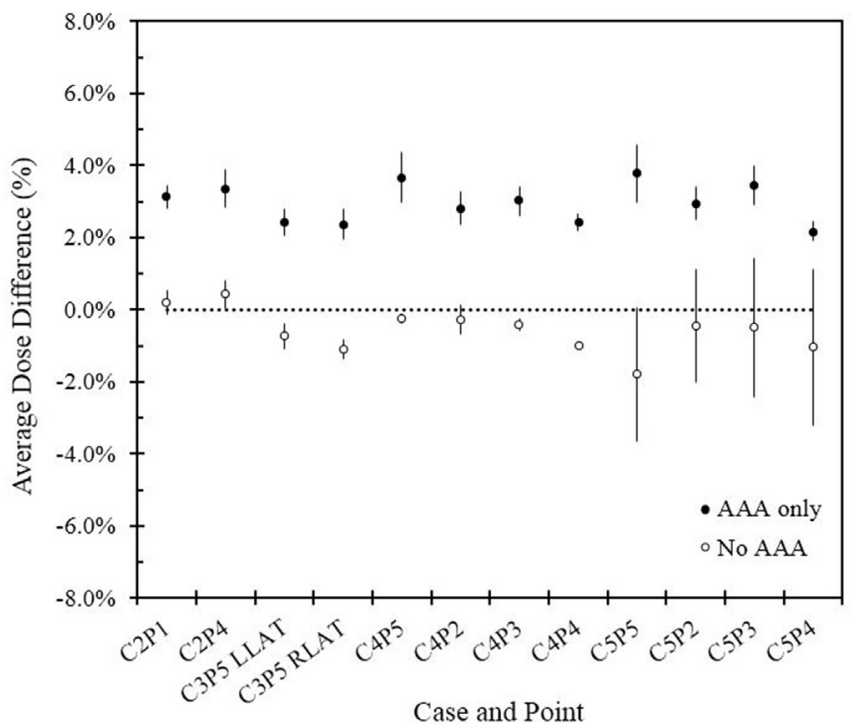

Figure 4. Measurement beam specific average Level III audit result for individual points downstream from the lung material. The closed circles show the results for only the AAA algorithm and the open circles shows the results for all other algorithms. Differences are calculated and normalized as before. Error bars represent the standard error of the mean. employing the AAA algorithm. Referring to Table 2 and Fig. 2, the cases that meet these conditions are Cases 11 to 15 , points 1 and 3 . The results from Case 16, the asymmetric field with $60^{\circ}$ wedge are not included due to the inability of some treatment planning system and linac combinations to calculate this field accurately [18]. In Fig. 7, each point shows the average dose discrepancy between measured and planned doses, relative to the dose difference of the reference Case 2 Point 1 to eliminate daily linac output and individual facility dosimetry. The distinct $3.0 \%$ offset present in the Level III audit results (Figs. 3 and 4 ) is not seen for the Level II audit, with the average discrepancy for AAA and other algorithms with the mean and standard deviation of the average differences for AAA and all other algorithms found to be $0.2 \pm 0.7 \%$ and $-0.7 \pm 0.9 \%$ respectively.

\section{Discussion}

The data presented in this manuscript demonstrates a peculiarity of the AAA algorithm, as implemented in the Varian Eclipse TPS. For curved, 'patient realistic' inhomogeneities such as those

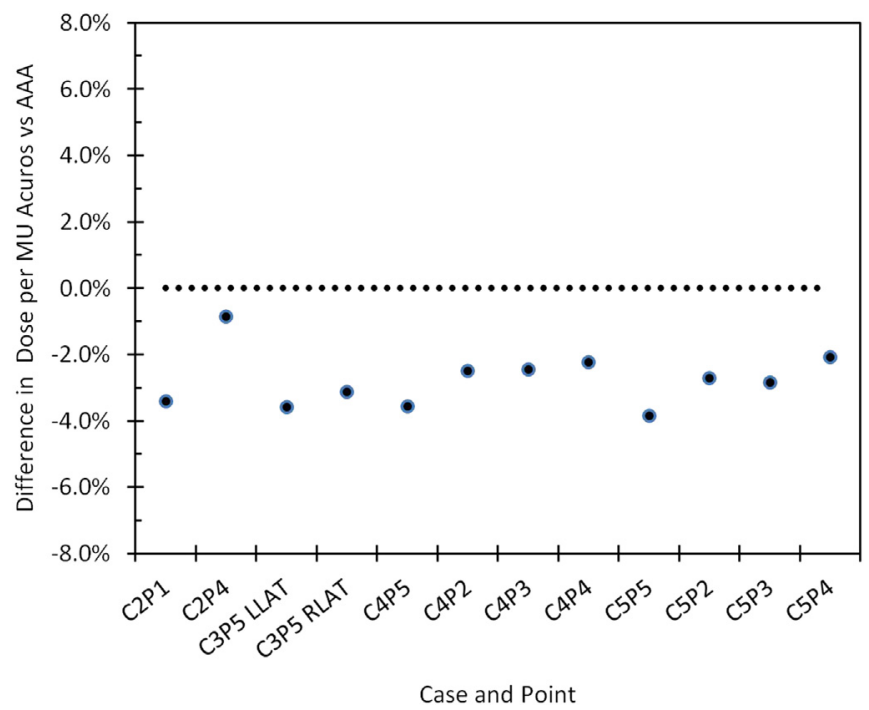

Figure 5. Difference in dose per MU for the points of level III audits when calculated using the Acuros (dose to water) vs the AAA algorithm. 

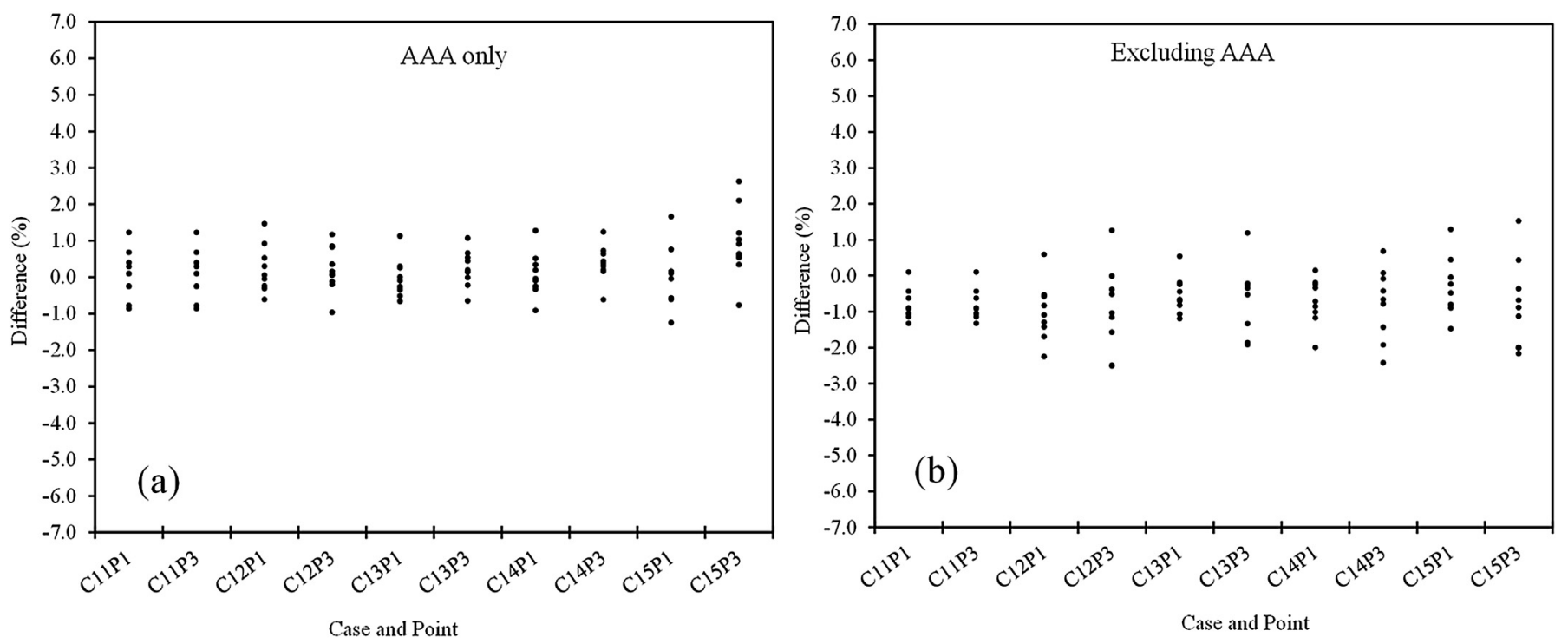

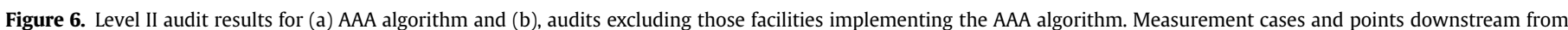

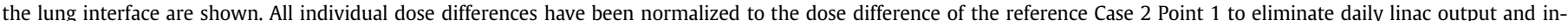
dividual facility dosimetry deviations. The mean and standard deviation of the average differences for AAA and all other algorithms is $0.2 \pm 0.7 \%$ and $-0.7 \pm 0.9 \%$ respectively.

present in the CIRS thorax phantom, the AAA algorithm consistently calculates a higher dose than is measured. On average, this difference amounts to approximately $3.0 \%$ when the measurement point is downstream from the lung inhomogeneity in tissue equivalent material. Other algorithms, tested in the same manner do not show this behaviour. The systematic discrepancy of the AAA algorithm was not found when the phantom and the inhomogeneity were of a rectilinear slab geometry. Here, on average, the AAA algorithm calculated the dose actually more accurately, though not significantly higher than the average of the other algorithms $(0.2 \pm 0.7 \%$ and $-0.7 \pm 0.9 \%$ mean $\pm S D$, respectively).

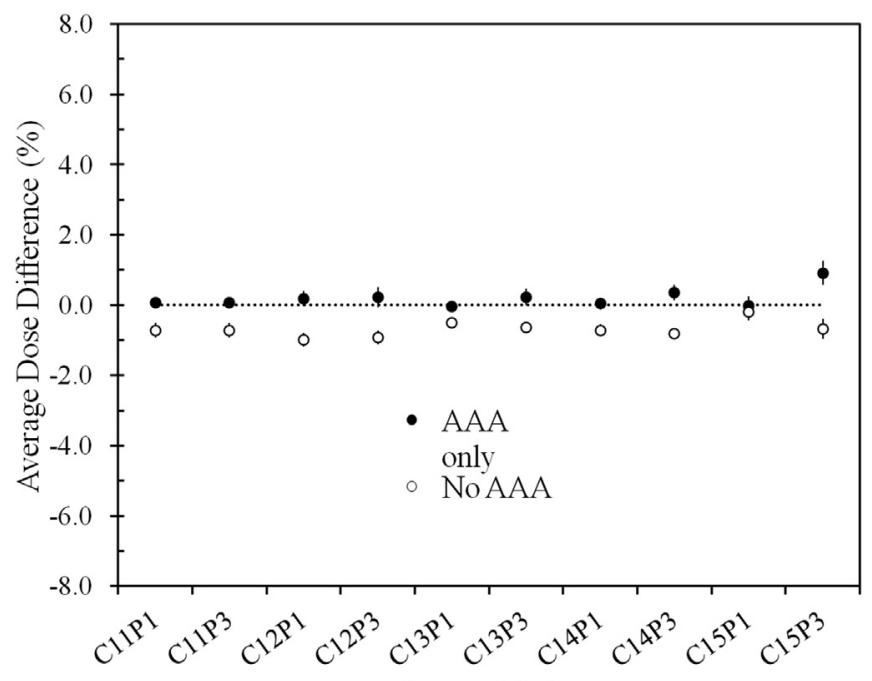

Case and Point

Figure 7. The average Level II audit results (Figure 5) for the centre of field points for individual cases behind lung. The closed circles show the results for only the AAA algorithm and the open circles shows the results for all other algorithms. All individual dose differences have been normalized to the dose difference of the reference Case 2 Point 1 to eliminate daily linac output and individual facility dosimetry deviations. Error bars represent the standard error of the mean.
This could potentially be a problem if commissioning measurements for a Varian Eclipse TPS are done with rectilinear phantoms. In end-to-end tests, which are generally also performed as part of the commissioning, any algorithm rooted deviations might be diminished by other small deviations or might be within the larger allowed tolerances for such tests [24].

There are a number of experimental studies for which the AAA algorithm has been shown to be accurate in both homogeneous and heterogeneous geometries [21,25-27]. Ronde et al. [28] investigated two-dimensional dose distributions for a variety of different field configurations in solid water with a 2D array of ion chambers, as well as patient specific treatment plans in a thorax lung phantom with Gafchromic films. Their study found that the AAA algorithm was superior to the $\mathrm{PBC}$ algorithm in heterogeneous media, especially for $15 \mathrm{MV}$. Furthermore, when measuring in a thorax phantom, the deviations between planned and measured doses in lung and mediastinum regions was found to be less than their specified criteria of $3 \%$ in dose and $3 \mathrm{~mm}$ in distance-to-agreement (DTA) respectively.

Van Esch et al. [4] tested the accuracy of AAA for different field sizes (symmetric and asymmetric) for open fields, wedged fields, and static and dynamic multi-leaf collimation fields. Measurements were performed on homogeneous (water equivalent), phantoms containing cork inhomogeneities, and on the thorax of an anthropomorphic phantom. Their measurements demonstrated that at the interface between solid water and cork, dose profiles showed a better agreement with AAA compared to Pencil Beam. Depth dose curves in the cork were also improved substantially with AAA when compared to Pencil Beam models. For the thorax phantom dose measurements, the agreement between planned and measured doses was found to be mostly within $5 \%$. This result is not in disagreement with the results presented in Fig. 3, though the systematic offset of $3 \%$ was not reported, possibly due to the assessment criteria of $5 \%$.

Bragg et al. [29] compared predicted and measured dose distributions with ion chamber and film measurements in homogeneous and inhomogeneous phantoms, with simple and complex fields. The effect of inhomogeneities was investigated using a range of phantoms constructed of water, bone and lung substitute materials. Simulated treatment plans were also 
delivered to an anthropomorphic phantom and the delivered doses compared to the doses predicted by the Anisotropic Analytical Algorithm. For homogeneous phantoms, Bragg et al. found that the agreement was within $2 \%$ and $2 \mathrm{~mm}$ DTA in most instances. When heterogeneities were present, discrepancies between measured and planned doses were generally found to be within $2.5 \%$ for slab and anthropomorphic phantoms respectively.

In contrast to the above studies, Robinson [30] evaluated the ability of the AAA algorithm to accurately account for the presence of inhomogeneities in simple geometries by comparing calculated and measured doses. The study used a planar geometry phantom consisting of upper and lower layers of Solid Water separated by a heterogeneity region of variable thickness modelled in Eclipse. The goal of $2 \%$ accuracy in correcting for heterogeneities, as set out by the American Association of Physicists in Medicine Task Group 65 [31] was used as the criteria for evaluation. In general, over all nonunit density materials examined, the AAA was found to overpredict (number of MUs for actual treatment delivery would result in a dose deficiency) dose beyond low-density regions and in many cases, the deviation between the AAA and experimental results exceeds the Task Group 65 target of $2 \%$. The source of these deviations was speculated to arise from an inability of the AAA algorithm to correctly account for altered attenuation along primary ray paths.

National/international audit programs to combine multi-centre data and can thus leverage a statistical power to identify correlations which are invisible within, or to, a single facility. The dosimetric discrepancy identified and quantified by the ACDS while mentioned in the published literature, has gone unnoticed or underestimated by individual facilities. These results reinforce the case that independent external dosimetric auditing is an invaluable tool for improving, and validating, the accuracy of radiation therapy. Kry et al. [32] and the Imaging and Radiation Oncology Core (IROC - Houston) group, further demonstrate the utility and value of such multi-centre auditing. Their study compared institution patient-specific IMRT QA plans to the results of their independent credentialing using a head phantom. The study found that in-house IMRT QA failed to detect unacceptable plan delivery as measured independently in an IROC head and neck phantom and concluded that IMRT QA was not a reasonable replacement for a credentialing (audit) phantom irradiation. In a similar study to the work presented here, Kry et al. [24] used a thorax phantom for a large $(n=221$ different institutions, 304 irradiations) credentialing study that looked at the impact of dose calculation algorithm and their accuracy when accounting for heterogeneities. Measured doses were compared to calculated doses for a lung target and analysed as a function of calculation algorithm. Their results showed that the AAA algorithm as well as convolution-superposition algorithms overestimated the dose that was delivered to the target by $3.7 \%$ on average. Though direct comparisons cannot be made between the results presented here owing to the nature of the audits performed (in lesion surrounded by lung, versus in tissue downstream from lung), it is probable that such a systematic discrepancy may not have been uncovered were it not for the multi-centre audit.

A large European study by Gershkevitsh et al. [33] conducted TPS audits in 60 radiotherapy centres using the TECDOC 1583 methodology also employed in the Level III audit presented here. In total, 190 data sets (combination of algorithm and beam quality) were collected and analysed. Their study found that dosimetry problems requiring interventions were discovered in about $10 \%$ of datasets and deficient beam modelling in TPSs was discovered in a number of cases. Like the RPC and ACDS audit programs, the IAEA European audit contributed to achieving a better understanding of the performance of TPSs and helped to resolve issues related to imaging, dosimetry and treatment planning. Furthermore, their study also found similar discrepancies for the Varian AAA algorithm however the results were attributed to input beam data and beam modelling. Similar European and South American audits have also been reported and shown to be effective [34,35].

The results in this work have not been reported by previous studies, employing similar anthropomorphic phantoms. The possible reasons for this are the use of wider overall dose delivery tolerances of 5\% [36], resulting in measurements with 3\% discrepancy still passing the acceptance criteria. The Level III audits in this work are conducted on onsite audits using directly PSL calibrated Farmer type ionization chambers and electrometers resulting in low measurement uncertainties and allowing for tight action-level tolerances. The contribution of other factors, such as incorrect beam modelling and input data as reported in Gershkevitsh et al. [33] may also have contributed to the oversight of the systematic nature of the discrepancy.

\section{Conclusion}

This work has demonstrated a systematic underdosing that occurs when the AAA algorithm is compared to measurements in the mediastinum region of an anthropomorphic thorax phantom, with lateral beams passing through lung. This systematic offset, which was absent for identical comparisons with other algorithms, results in an average discrepancy of $3 \%$ between planned and measured doses. This result, common amongst separate institutions using the same dose calculation algorithm and parameters, may have otherwise been overlooked by individual facilities, and was underestimated by others. An independent external auditing process was required to identify the dosimetric issue.

The ACDS Level III audit (as well as the Level II) has an out-oftolerance level of $5 \%$ and discrepancies between measured and planned doses of $3 \%$ did not necessarily result in out-of-tolerance audit outcomes. However, the systematic discrepancy due to the AAA algorithm, when compounded with other errors in the treatment planning and delivery chain more often resulted in discrepancies outside the optimal level $(\leq 3.3 \%$ difference between measured and planned dose) in comparison to other algorithms for the same measurements.

The potential implication of this finding in clinical scenarios is lower tumour control probability for lesions downstream from lung, for example, lung and mediastinal tumours when the AAA algorithm is used. Additionally, other sources of uncertainty in the radiotherapy treatment chain and dosimetry may compound together to result in a clinically relevant dose discrepancy between what was planned and actually delivered to the patient. Conversely, other sources may compensate for the calculation discrepancy, hiding the issue. We speculate that the reason for the results presented in this work is that AAA uses Gaussian functions to describe the mean heterogeneous effect in just four lateral directions, instead of over $4 \pi$ as implemented in the Acuros XB algorithm. Therefore, a solution for facilities with the Eclipse TPS is to consider the use of the Acuros XB dose calculation algorithm, which appears to generally handle most of the situations investigated with the ACDS Level III audits more accurately.

\section{Acknowledgements}

The Australian Clinical Dosimetry Service is a joint initiative between the Department of Health and the Australian Radiation Protection and Nuclear Safety Agency. 


\section{References}

[1] Ahnesjö A, Aspradakis M. Dose calculations for external photon beams in radiotherapy. Phys Med Biol 1999;44:R99.

[2] Moyed Miften K, Wiesmeyer Mark, Monthofer Suzanne, Ken. “Implementation of FFT convolution and multigrid superposition models in the FOCUS RTP system. Phys Med Biol 2000;45:817.

[3] Ahnesjo A. Collapsed cone convolution of radiant energy for photon dose calculation in heterogeneous media. Med Phys Jul-Aug 1989;16:577-92.

[4] Van Esch A, Tillikainen L, Pyykkonen J, Tenhunen M, Helminen H, Siljamaki S, et al. Testing of the analytical anisotropic algorithm for photon dose calculation. Med Phys Nov 2006;33:4130-48.

[5] Bortfeld T, Schlegel W, Rhein B. Decomposition of pencil beam kernels for fast dose calculations in three-dimensional treatment planning. Med Phys 1993;20:311-8.

[6] Zhang P, Simon A, De Crevoisier R, Haigron P, Nassef MH, Li B, et al. A new pencil beam model for photon dose calculations in heterogeneous media. Phys Medica 2014;30:765-73.

[7] Irvine C, Morgan A, Crellin A, Nisbet A, Beange I. The clinical implications of the collapsed cone planning algorithm. Clin Oncol 4/2004;16:148-54.

[8] Tommy Knöös L, Wieslander Elinore, Cozzi Luca, Brink Carsten, Fogliata Antonella, Albers Dirk, et al. Comparison of dose calculation algorithms for treatment planning in external photon beam therapy for clinical situations. Phys Med Biol 2006;51:5785.

[9] Fogliata A, Vanetti E, Albers D, Brink C, Clivio A, Knöös T, et al. On the dosimetric behaviour of photon dose calculation algorithms in the presence of simple geometric heterogeneities: comparison with Monte Carlo calculations. Phys Med Biol 2007;52:1363.

[10] Vanderstraeten B, Reynaert N, Paelinck L, Madani I, De Wagter C, De Gersem W, et al. Accuracy of patient dose calculation for lung IMRT: a comparison of Monte Carlo, convolution/superposition, and pencil beam computations. Med Phys 2006;33:3149-58.

[11] Knoos T, Ahnesjo A, Nilsson P, Weber L. Limitations of a pencil beam approach to photon dose calculations in lung tissue. Phys Med Biol 1995;40:1411.

[12] Francescon P, Cavedon C, Reccanello S, Cora S. Photon dose calculation of a three-dimensional treatment planning system compared to the Monte Carlo code BEAM. Med Phys 2000;27:1579-87.

[13] Carrasco P, Jornet N, Duch MA, Weber L, Ginjaume M, Eudaldo T, et al. Comparison of dose calculation algorithms in phantoms with lung equivalent heterogeneities under conditions of lateral electronic disequilibrium. Med Phys 2004;31:2899-911.

[14] H. Siantar CL, Walling RS, Daly TP, Faddegon B, Albright N, et al. Description and dosimetric verification of the Peregrine Monte Carlo dose calculation system for photon beams incident on a water phantom. Med Phys 2001;28: $1322-37$.

[15] Martens C, Reynaert N, De Wagter C, Nilsson P, Coghe M, Palmans H, et al. Underdosage of the upper-airway mucosa for small fields as used in intensitymodulated radiation therapy: a comparison between radiochromic film measurements, Monte Carlo simulations, and collapsed cone convolution calculations. Med Phys 2002;29:1528-35.

[16] Williams I, Kenny J, Lye J, Lehmann J, Dunn L, Kron T. The Australian Clinical Dosimetry Service: a commentary on the first 18 months. Australas Phys Eng Sci Med 2012:1-5.

[17] Lye J, Kenny J, Lehmann J, Dunn L, Kron T, Alves A, et al. "A 2D ion chamber array audit of wedged and asymmetric fields in an inhomogeneous lung phantom. Med Phys 2014;41:101712. 1-11.
[18] Lehmann J, Kenny J, Lye J, Dunn L, Williams I. Trust, but verify-accuracy of clinical commercial radiation treatment planning systems. J Phys Conf Ser 2014;489:012094.

[19] Lu L. Dose calculation algorithms in external beam photon radiation therapy. Int J Cancer Ther Oncol 2013;1.

[20] Ulmer W, Pyyry J, Kaissl W. A 3D photon superposition/convolution algorithm and its foundation on results of Monte Carlo calculations. Phys Med Biol 2005;50:1767.

[21] Gagne IM, Zavgorodni S. Evaluation of the analytical anisotropic algorithm (AAA) in an extreme water-lung interface phantom using Monte Carlo dose calculations. J Appl Clin Med Phys 2007;8.

[22] Sievinen J, Ulmer W, Kaissl W. AAA photon dose calculation in eclipse," varian documentation RAD B, vol. 7170; 2005. p. 2005.

[23] IAEA. TecDoc 1583: commissioning of radiotherapy treatment planning systems: testing for typical external beam treatment techniques. Vienna: International Atomic Energy Agency; 2008.

[24] Kry SF, Alvarez P, Molineu A, Amador C, Galvin J, Followill DS. Algorithms used in heterogeneous dose calculations show systematic differences as measured with the radiological physics center's anthropomorphic thorax phantom used for RTOG credentialing. Int J Radiat Oncol* Biol* Phys 2013;85:e95-100.

[25] Hussain A, Villarreal-Barajas E, Brown D, Dunscombe P. Validation of the eclipse AAA algorithm at extended SSD. J Appl Clin Med Phys 2010;11:3213.

[26] Breitman K, Rathee S, Newcomb C, Murray B, Robinson D, Field C, et al. Experimental validation of the eclipse AAA algorithm. J Appl Clin Med Phys 2007;8:76-92.

[27] Kan MW, Leung LH, So RW, Yu PK. Experimental verification of the acuros XB and AAA dose calculation adjacent to heterogeneous media for IMRT and rapid arc of nasopharygeal carcinoma. Med Phys Mar 2013;40:031714.

[28] Ronde HS, Hoffmann L. Validation of Varian's AAA algorithm with focus on lung treatments. Acta Oncol 2009;48:209-15.

[29] Bragg CM, Wingate K, Conway J. Clinical implications of the anisotropic analytical algorithm for IMRT treatment planning and verification. Radiother Oncol 2/2008;86:276-84.

[30] Robinson D. Inhomogeneity correction and the analytic anisotropic algorithm. J Appl Clin Med Phys 2008;9:2786.

[31] AAPM. American Association of Physicists in Medicine (AAPM) task group 65. Madison (WI) Tissue Inhomogeneity Correct Megavoltage Photon Beams 2004.

[32] Kry SF, Molineu A, Kerns JR, Faught AM, Huang JY, Pulliam KB, et al. "Institutional patient-specific IMRT QA does not predict unacceptable plan delivery. Int J Radiat Oncol Biol Phys Dec 1 2014;90:1195-201.

[33] Gershkevitsh E, Pesznyak C, Petrovic B, Grezdo J, Chelminski K, do Carmo Lopes M, et al. Dosimetric inter-institutional comparison in European radiotherapy centres: results of IAEA supported treatment planning system audit. Acta Oncol 2014;53:628-36.

[34] Lopes MC, Cavaco A, Jacob K, Madureira L, Germano S, Faustino S, et al. Treatment planning systems dosimetry auditing project in Portugal. Phys Medica 2014;30:96-103.

[35] Samper J.L., Alfonso-Laguardia R., Yip F.G., Larrinaga-Cortina E., Morales-Lopez J.L., and Silvestre-Patallo I., "Use of an anthropomorphic phantom to improve the external beam quality audits in radiotherapy".

[36] ICRU. Determination of absorbed dose in a patient irradiated by beams of X or gamma rays in radiotherapy procedures. In: Reprint: International Commission on Radiation Units and Measurements; 1991. 